\title{
A Machine Learning-Based Algorithm for Processing Massive Data Collected from the Mechanical Components of Movable Bridges
}

\author{
F. Necati Catbas ${ }^{1}$ and Masoud Malekzadeh ${ }^{1,2}$ \\ ${ }^{1}$ University of Central Florida (catbas@ucf.edu) \\ ${ }^{2}$ Metal Fatigue Solutions (m.malekzadeh@knights.ucf.edu )
}

\begin{abstract}
This paper presents a machine learning algorithm for processing of massive data collected from the mechanical components of movable bridges. The proposed approach consists of training and monitoring phases. The training phase was focused on the extracting statistical features and conducting cross correlation analysis (CCA) and robust regression analysis (RRA). The monitoring phase included tracking of errors associated with the derived models. The main goal was to analyze the efficiency of the developed system for health monitoring of the bridge mechanical components such as gearbox, motor and rack and pinion. To this aim, Sunrise Movable Bridge in Ft. Lauderdale, Florida was selected and instrumented. A comprehensive database was collected from the sensors installed on the mechanical and structural components of the Sunrise Bridge for about 4 years. The collected data were utilized to assess the performance of the algorithm under baseline and different common damage scenarios. Based on the results, the proposed health monitoring system has a satisfactory performance for the detection of the damage scenarios caused by leakage and lack of sufficient oil in gearbox, as well as bolt removal from rack and pinion. The introduced algorithm can be regarded as a valuable tool for the management and interpretation of the massive (big) data collected for structural health monitoring (SHM) of movable bridges.
\end{abstract}

Keyword: Maintenance Monitoring, Machine-Learning, Massive SHM data, Robust Regression Analysis, Movable Bridge, Damage Detection

\section{INTRODUCTION}

Obtaining reliable and timely assessment of bridge condition, performance and safety of bridges over their long service lives represents a considerable challenge for bridge owners and engineers [1]. The ability to quantitatively characterize existing bridges may lead to more cost-effective and efficient maintenance management decisions, and more robust evaluation of structures. Currently, the long-term performance and condition of most bridges are evaluated on the basis of biennial visual inspections [2]. Visual inspection data are inherently qualitative and is subject to other important limitations that can hamper their effectiveness for assessing bridge condition and safety. A study by the FHWA on the reliability of visual inspection revealed many of the uncertainties associated with this assessment approach [3]. Structural Health Monitoring (SHM) is an emerging approach that has gained significant attention because of its promise to enable more quantitative, reliable and timely assessments of bridges than using only visual inspections [4]. 
The first step toward SHM is to design a network of sensors that can continuously collect information at the critical locations of a given structure [5]. However, lack of cost-effective and reliable instrumentation devices (including both sensors and data acquisition systems) has been a major challenge for the practical implementation of SHM methodology until a decade ago. Nevertheless, thanks to the significant advances and improvements in the field of electrical engineering and sensor technology over the past decade, collecting SHM data over long-term is no longer a big challenge for the researchers and engineers.

Having access to reliable and non-expensive sensors and data acquisition systems provides an opportunity to move toward more efficient data collection for long-term monitoring of civil and mechanical infrastructures. This revolution has shifted the focus of the researchers from the sensing and instrumentation to the SHM data interpretation area [6-7]. Indeed, investing a significant amount of budget for monitoring a structure over long period of time eventually results in massive (big) amount of SHM data that has to be well-interpreted otherwise it may not have much value [8-9]. Therefore, novel sensing technologies backed up with advanced and effective data interpretation methodologies will be an invaluable decision-support tool for

Inherently, the data interpretation techniques that are utilized for SHM are classified as either parametric (model-based) or non-parametric (model-free or data-driven) approaches. Basically, through the parametric approach an initial mathematical model of structure is developed and updated with the measurements. Once the calibrated model is identified it is utilized for predicting the non-measurable structural responses.

There are some responses or attributes of structure that can be measured by means of sensors such as strain, displacement, acceleration, etc. In most cases, however, these types of responses are not directly carrying the sort of information that can be beneficial for identifying the healthy status of structure. Instead, other responses are of interest such as load carrying, capacity, reliability index and, etc. The solution to this challenge is referred as structural identification [11]. In fact, structural identification can be viewed as translation of "raw" measured parameters into "actionable" information [12-13].

Alternatively, the non-parametric approach relies on statistical analysis of the data and deviation from a pre-established threshold limit [14-18]. In fact, statistical techniques including data mining and machine learning methods are used to create a baseline condition of the structure and any variation from that may be considered and reported as abnormal behavior [19-20]. These techniques are preferred in the circumstances in which creating a behavioral model is either time consuming or expensive. Being independent of the model information turns the non-parametric approach into an ideal one for continuous, efficient and automated monitoring. As a consequence, the focus of this study is devoted to design a non-parametric algorithm, which can be used for continuous processing of 'big' SHM data collected from critical mechanical components of movable bridges.

89 


\subsection{Objective and Scope}

The objective of this study is to propose a new approach for long-term operational monitoring of the movable bridges. Long-term vibration data has been collected for over 4 years from the Sunrise Movable Bridge in Ft. Lauderdale, Florida, USA. Data has been collected using accelerometers that were installed on the mechanical components including gearbox, motor and rack and pinion [1]. The vibration data has been collected, for over 4 years, during the opening phase of the movable bridge where the mechanical components start operating and supposedly experiencing the highest level of vibrations.

A framework for data analysis is designed incorporating Robust Regression Analysis (RRA). The proposed unsupervised algorithm consist of two individual sections including training and monitoring phases. The underlying distribution of baseline condition is learnt throughout the training phase whereas the monitoring phase is designed to process the real-time data and to detect any possible abnormal behavior by comparing the results with the established thresholds. Data from both healthy and damaged conditions are needed to thoroughly evaluate the efficacy of the proposed algorithm. Yet, this is usually challenging due to the unavailability of the damaged data from the real-life civil infrastructures. In this study, the common and typical damage scenarios associated with critical mechanical components of a movable bridge are identified and simulated temporarily on the bridge using the valuable feedback from the Department of Transportation (DOT) engineers and maintenance personnel.

Therefore, the main contribution of this study is to introduce an effective approach based on RRA algorithm to extract important information from massive amount of data collected from movable bridges. The main objective is to timely detect any possible abnormal behavior of the movable bridges using the specially designed monitored system. Also, another unique aspect of this study is the monitored structure and long-term data (big data) utilized in this study to verify the efficiency of the algorithm. As mentioned earlier, it is usually very rare to have access to the damaged SHM data, in particular from a unique real-life structure such as the movable bridge. In this study, data from both baseline and damaged conditions are utilized to test and verify the proposed approach.

As a result, the layout of this paper is presented as follows. First, the SHM system at the Sunrise Blvd. Bridge is demonstrated with an emphasis on the mechanical components including the gearbox, motor and rack and pinion. Next, the simulated damages on the gearbox and rack and pinion are described. Later, a brief summary of the regression analysis with a particular focus on the robust regression analysis is presented. Finally, efficiency of the proposed algorithm is investigated using the collected massive (big) SHM data from the Sunrise Bridge. 


\section{STRUCTURAL HEALTH MONITORING OF THE SUNRISE MOVABLE BRIDGE}

For static structures, monitoring of structural components is usually the only concern for maintenance, safety and operation. However, monitoring of mechanical and electrical components is equally important for movable bridges. Bridge opening and closing operations induce additional stress on the structural and mechanical components of a movable bridge due to the generated mechanical and dynamic forces. Therefore, a properly designed monitoring system for a movable bridge should consider all structural, mechanical and electrical components of a movable bridge.

140
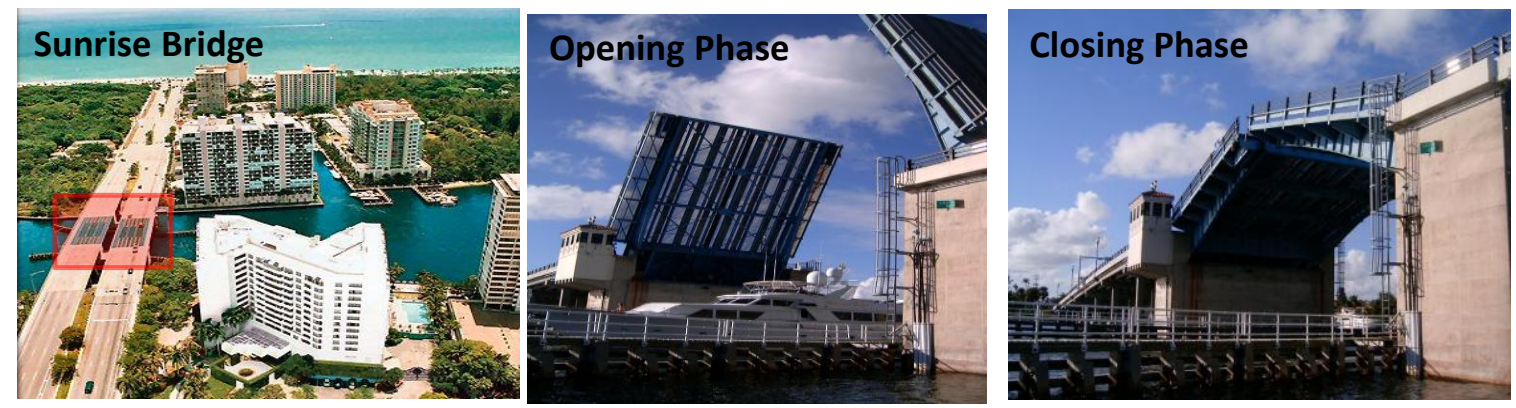

Figure 1- Sunrise Bridge in Ft. Lauderdale, Florida

The SHM data that is used in this study is a part of a comprehensive database from more than 200 channels of sensors to track and monitor the performance of critical structural, mechanical and electrical components of the movable bridge (Figure 1) [1]. The readers are referred to the main report for detailed information and discussion [21]. This paper is concentrated on the mechanical component monitoring of the project where the objective is to monitor the conditions of mechanical components for maintenance purposes.

The most important mechanical components of a movable bridge are the gearbox, motor, rack and pinion, shaft, open gear and trunnion. Locations of these components are schematically illustrated in Figure 2. In this study, the corresponding data sets from the gearbox, motor and rack and pinion are utilized. Herein, a brief discussion on each of these components including their 

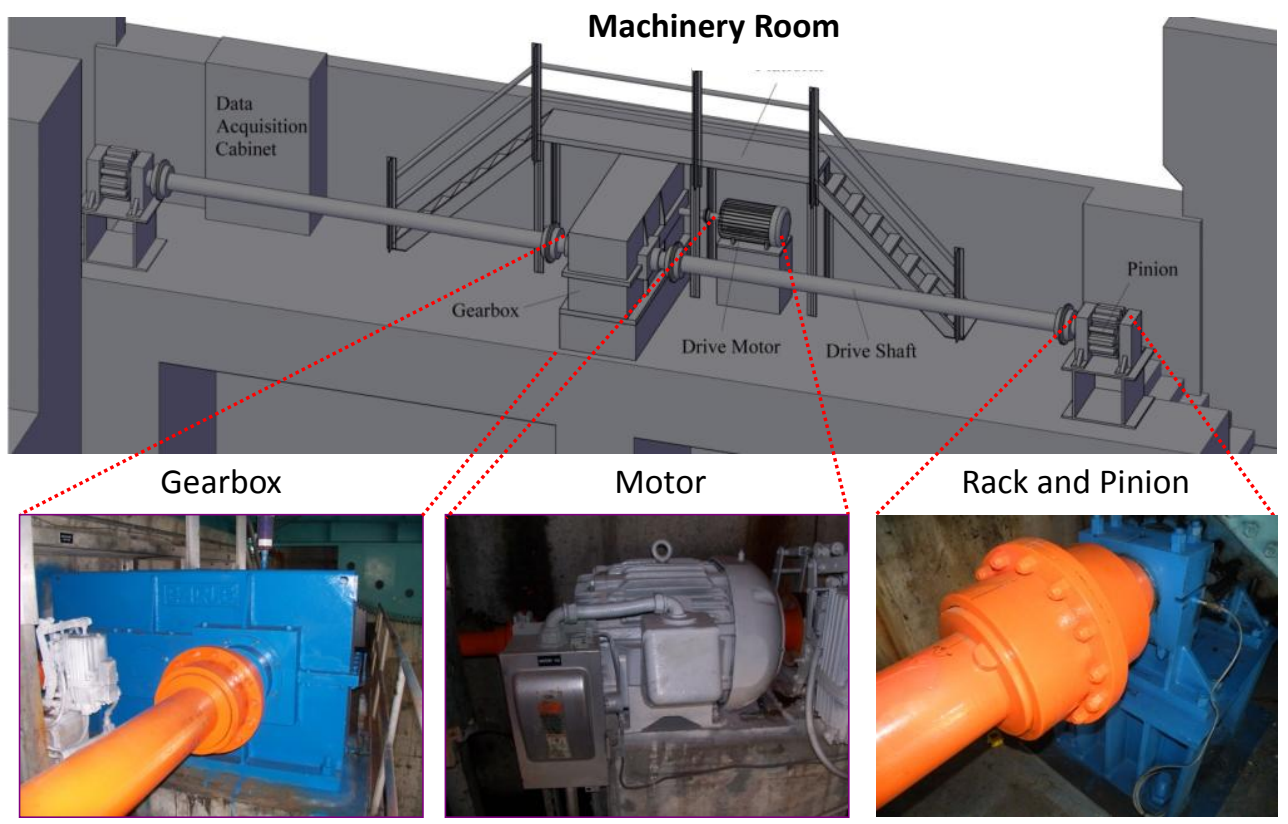

Figure 2- Machinery Room for the mechanical components of the Sunrise Bridge

\subsection{Critical Mechanical Components}

\subsubsection{Gearbox}

The gearbox contains the assembly that transmits the torque generated by the motor to the shafts (Figure 3). When the gearbox experiences deterioration or lack of lubrication, some change in the vibration and sound characteristics could be noted during the bridge operation. Abnormal vibration is an indicator of wear in the gears. Oil viscosity is also an important parameter for proper functioning of the gearbox. Considering these issues, the monitoring system includes six accelerometers to measure the vibration of the gearbox during bridge opening and closing events. Furthermore, microphones are also installed in the vicinity of the gearbox to determine its acoustic signature for opening and closing events. In this study, as mentioned earlier, the data collected with accelerometers during opening phase is used for analysis (Figure. 3). 
175
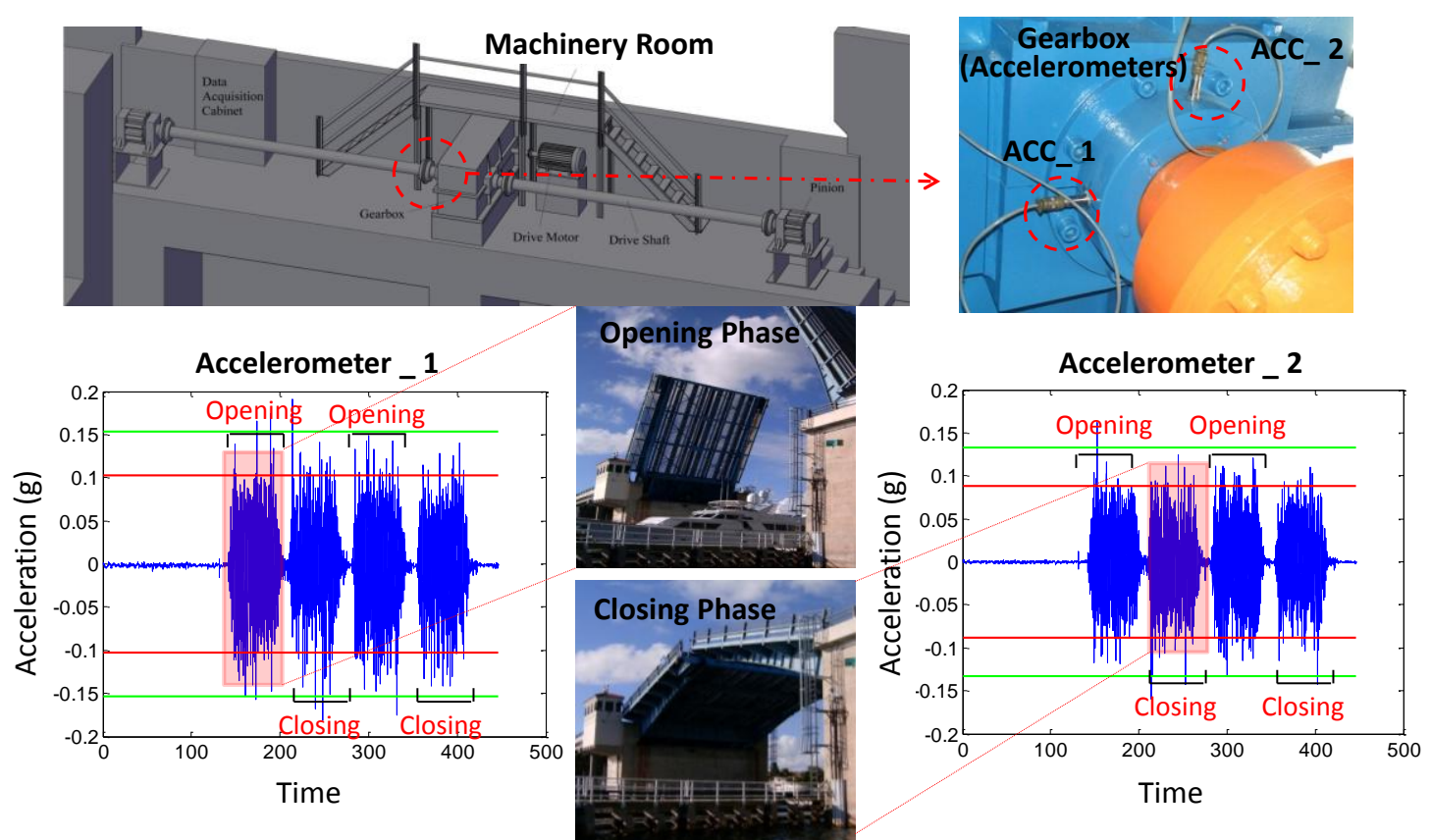

Figure 3- Sample measurement from the gearbox during opening and closing phases

\subsubsection{Electrical motor}

The electrical motors generate the torque required for the opening and closing of the bridge. Some of the indicators for improper functioning of the electrical motors are high amperage, high temperature, high vibration level and high revolution speed.
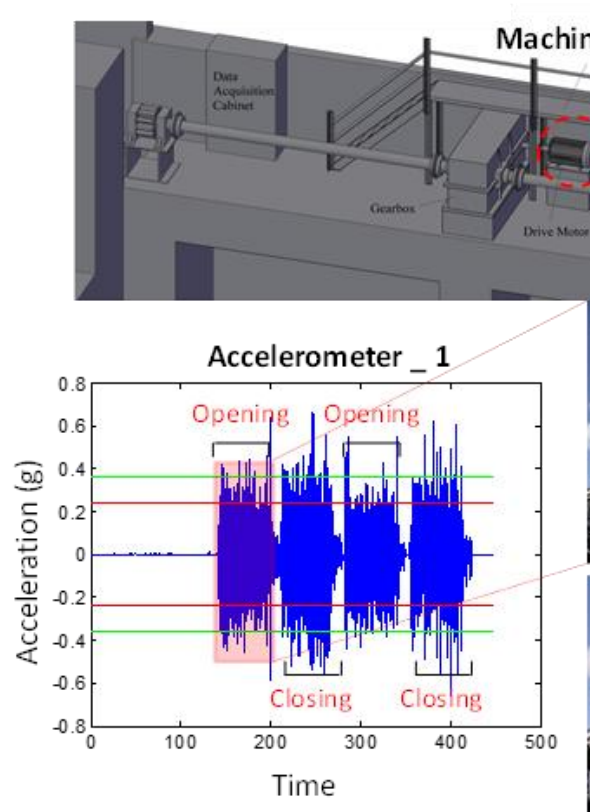

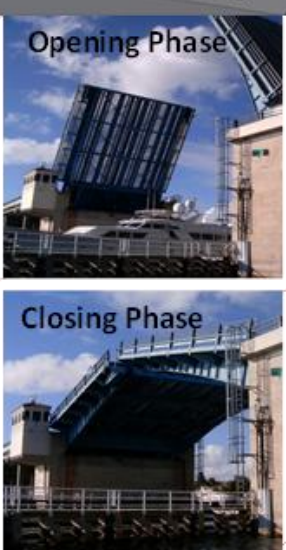

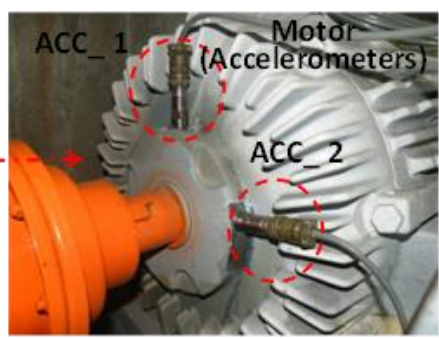

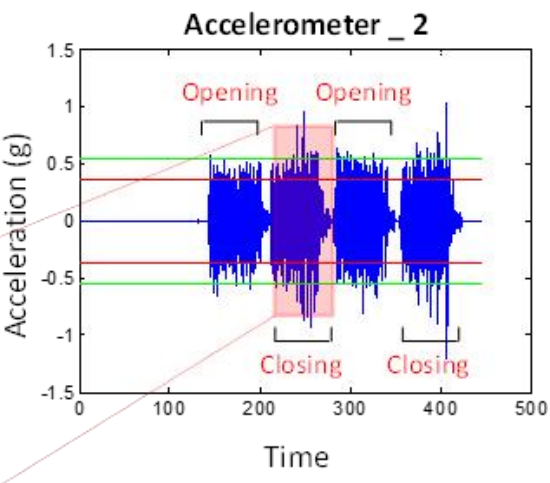


In order to monitor the issues related to the electrical motor, ampmeters have been added to the monitoring system to measure the amperage levels for each one of the electric motor phases. In addition, two accelerometers have been included to measure the vibration on the motor during the bridge openings and closings. Finally, infrared temperature sensors were utilized to monitor the temperature of the electrical motor (Figure 4).

Similar to the gearbox, the vibration data collected using the accelerometers is investigated to explore the maintenance condition of the electrical motor.

\subsubsection{Open Gear and Rack and Pinion}

200

The open gears are the main gears connected to the main girders at each leaf to receive the torque from the rack and pinion assembly. Corrosion due to lack of lubrication, excessive strain, out-ofplane rotation and misalignment are common problems for open gears. Another concern is loading sequence problems, which mean that the drive shafts begin rotation in delayed sequence. This has an adverse effect on the condition of the open gears, usually by causing impact loading. Routine maintenance is required on the gear teeth. If the gear teeth are not kept lubricated at all times, wear and corrosion due to grinding of the rack and the pinion will occur. To monitor the condition and maintenance needs of the open gears and rack and pinions, accelerometers were installed at the base of the rack and pinion to monitor its vibrations (Figure 5).

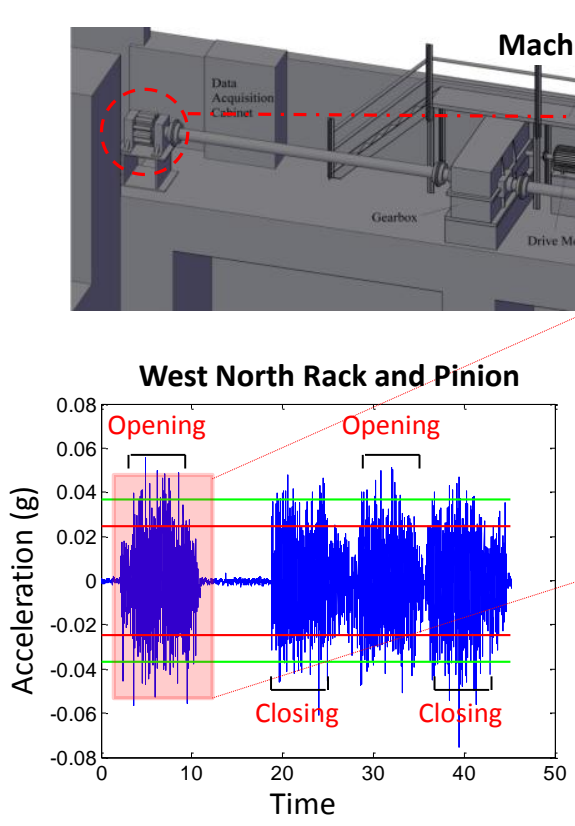

Figure 5- Sample measurement from the Rack and Pinion during opening and closing phases

\subsection{Field Tests with Artificially Induced Damages and Long Term Monitoring}


The long-term SHM data collected during this study can be utilized toward establishing criteria for movable bridge monitoring. This will assist operations by timely detecting maintenance effectiveness and needs. In order to meet the objective, field tests were conducted to establish thresholds for conditions that are critical for the maintenance and operation of the bridge. These conditions are referred to as "damage". In collaboration with DOT engineers, some of the most common mechanical maintenance problems are identified and subsequently implemented on the movable bridge to simulate such damage conditions. These common damages are discussed individually through the following sections.

\subsubsection{Damage Scenarios (typical abnormal behavior in mechanical components)}

\subsubsection{Gearbox oil removal}

The gearbox is equipped to provide the necessary amount of oil to the various gear meshes and bearings, thereby resulting in smooth and trouble free operation. The gearbox should be regularly checked for any leaks to see if the gearbox has adequate oil. In this project, the oil in the gearbox was partially removed to provide data corresponding to such an undesirable condition. Figure 6 (a) shows the removal of the oil from the gearbox. Only $25 \%$ of the oil was removed, and the effect of the oil reduction was monitored by six accelerometers attached to the gearbox during a few openings of the bridge spans.

\subsubsection{Rack and Pinion bolts removal}

The rack and pinion system is located between the shaft and the open gear; therefore, it can be considered a transmission zone for opening and closing operation forces. Therefore, it should be free from defects to ensure safe operation of the bridge. Here, the removal of bolts was the simulated damage scenario, and the effect of the absence of these bolts was monitored by two horizontal accelerometers at north and south part of the rack and pinion. Figure 6 (b) shows the Rack and Pinion with the removed bolts.

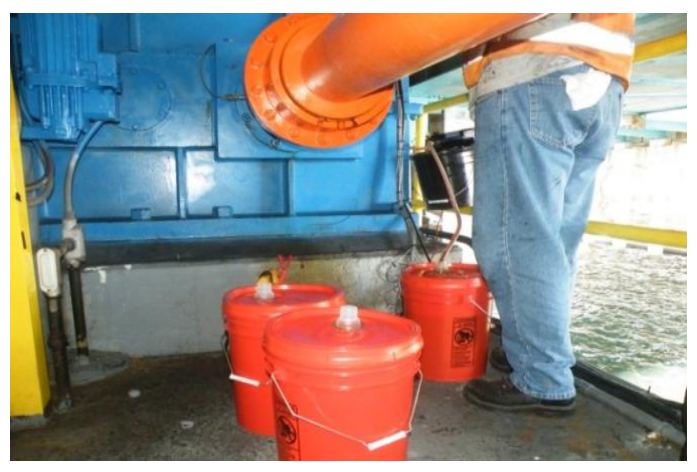

Extracting the Oil from the Gearbox
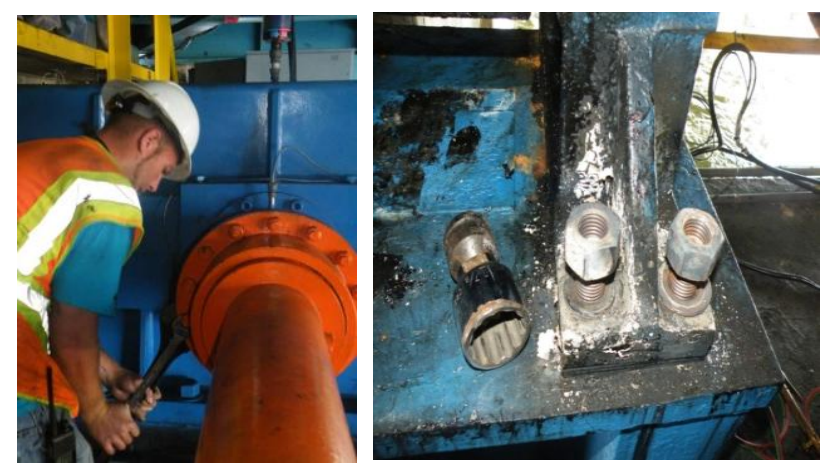

Removing the Bolts (Rack and Pinion)

Figure 6- Simulation of common critical maintenance issues. (a) Extracting oil from gearbox (b) Removing bolts from Rack and Pinion 


\section{NON-PARAMETRIC DAMAGE DETECTION ALGORITHM FOR MOVABLE BRIDGES}

The major activity of critical mechanical components takes place during the opining phase of the bridge where motor, gearbox and rack and pinion are all functioning together to open the bridge. As a result, the data acquisition system at the Sunrise Bridge has been programmed to start collecting data during the opening phases. Sunrise Bridge is being opened approximately 10 times per day to give marine traffic the right-of-way over the vehicular traffic. The size of a single data set collected from one accelerometer during each opining is equal to 16,000 data points, which accordingly results in $(16000 \times 10 \times 30)=4.8 \times 10^{6}$ data points per month. A simple calculation reveals that fact that $5.76 \times 10^{7}$ data points are collected from a single accelerometer over a year monitoring of Sunrise Bridge.

The data sets utilized for this study were collected between October 9, 2009 and May 3, 2013, which includes 5647 openings. Therefore, $9.0352 \times 10^{7}$ data points from each individual accelerometer are used in this study. Hence, an intelligent automated data processing algorithm is needed to manage and process this massive (big) amount of data and extract valuable maintenance related information. This big data has to be precisely analyzed and any possible abnormal behavior should be timely identified to mitigate any major functionality issue in movable bridges that may cause interruption in bridge operation.

Non-parametric techniques are independent of the model information and in most cases require minimum user interaction. For that reason, non-parametric techniques can potentially considered as an efficient solution for automated long-term monitoring. Robust Regression Analysis (RRA) is a non-parametric algorithm which has shown promising results in terms of long-term monitoring [15, 23]. Herein, a framework developed based on the RRA is proposed and utilized to process the massive (big) amount of SHM data collected over almost 4 years from the Sunrise Bridge. The efficiency of this algorithm, in term of detecting abnormal behavior in mechanical components, is tested by introducing some of the critical functionality issues to the Sunrise Bridge, as discussed in the previous sections.

\subsection{Robust Regression Analysis (RRA)}

Majority of the traditional regression techniques are based on least-square estimation and are very sensitive to their underlying assumptions. The main drawback associated with these techniques is their misleading results in the presence of outliers. These techniques provide favorable results as far as their underlying assumption are satisfied. As a result, ordinary regression techniques based on least squares are recognized to be not robust to the violation of their assumptions. This characteristic significantly reduces the efficiency of these methods for long-term SHM monitoring of civil infrastructures where data is usually contaminated with environmental effects (daily and seasonal), noise and outliers. To address the issue with underlying assumptions, robust regression methods are designed [23]. 
As discussed, regular regression techniques cannot be very effective for long-term monitoring due to their limitation when it comes to outliers. In order to address this shortcoming, a nonparametric damage detection algorithm, based on RRA, was proposed for continuous structural health monitoring applications [24-25].

However, the RRA damage detection algorithm used in these studies was designed to process continuous strain measurements. In this current paper, a new framework is proposed, based on the RRA, for continuous monitoring of acceleration data collected from critical mechanical components of movable bridges. The proposed algorithm is classified as unsupervised algorithm. For that reason, the algorithm should be provided with the data sets from the baseline (undamaged) condition to learn the corresponding underlying distribution. Once the confidence interval is established for the baseline condition, new measurements can be analyzed and compared to check with respect to the baseline thresholds. Therefore, prior to process the data with the proposed algorithm, data sets are divided into training and monitoring phases.

The successive steps for this algorithm are presented in Figure. 7 and Figure. 8 and will be further explained as following.

\section{I: Training phase}

Step1: Extract statistical features from acceleration data collected during opening phase, illustrated in Fig.7. As shown in Fig 7 and Fig 8, a matrix of features is created which includes selective statistical features of raw data. The statistical features used in this study are:

Note: $x_{\mathrm{i}}=\left\{x_{1}, x_{2} \ldots x_{\mathrm{n}}\right\}$ is a data set collected during $i^{\text {th }}$ opening.

1. Average of the ten largest values for each data set.

$$
\text { Feature } 1=F_{1}=\frac{1}{10} \sum_{n=1}^{10} x_{d_{n}}
$$

where $x_{d_{n}}=\left\{x_{d_{1}}, x_{d_{2}}, \ldots x_{d_{n}}\right\}$ is the result of sorting $x_{\mathrm{i}}$ in descending order.

2. Average of ten minimum values for each data set.

$$
\text { Feature } 2=F_{2}=\frac{1}{10} \sum_{n=1}^{10} x_{a_{n}}
$$

where $x_{a_{n}}=\left\{x_{a_{1}}, x_{a_{2}}, \ldots x_{a_{n}}\right\}$ is the result of sorting $x_{\mathrm{i}}$ in ascending order.

3. Standard deviation of each data set. 


$$
\text { Feature } 3=F_{3}=\sqrt{\frac{1}{n} \sum_{i=1}^{n}\left(x_{i}-\bar{X}\right)}
$$

330

$$
\text { Feature } 4=F_{4}=\sqrt{\frac{1}{n}\left(x_{1}{ }^{2}+x_{2}{ }^{2}+\cdots+x_{n}{ }^{2}\right)}
$$

4. Root mean square of each data set.

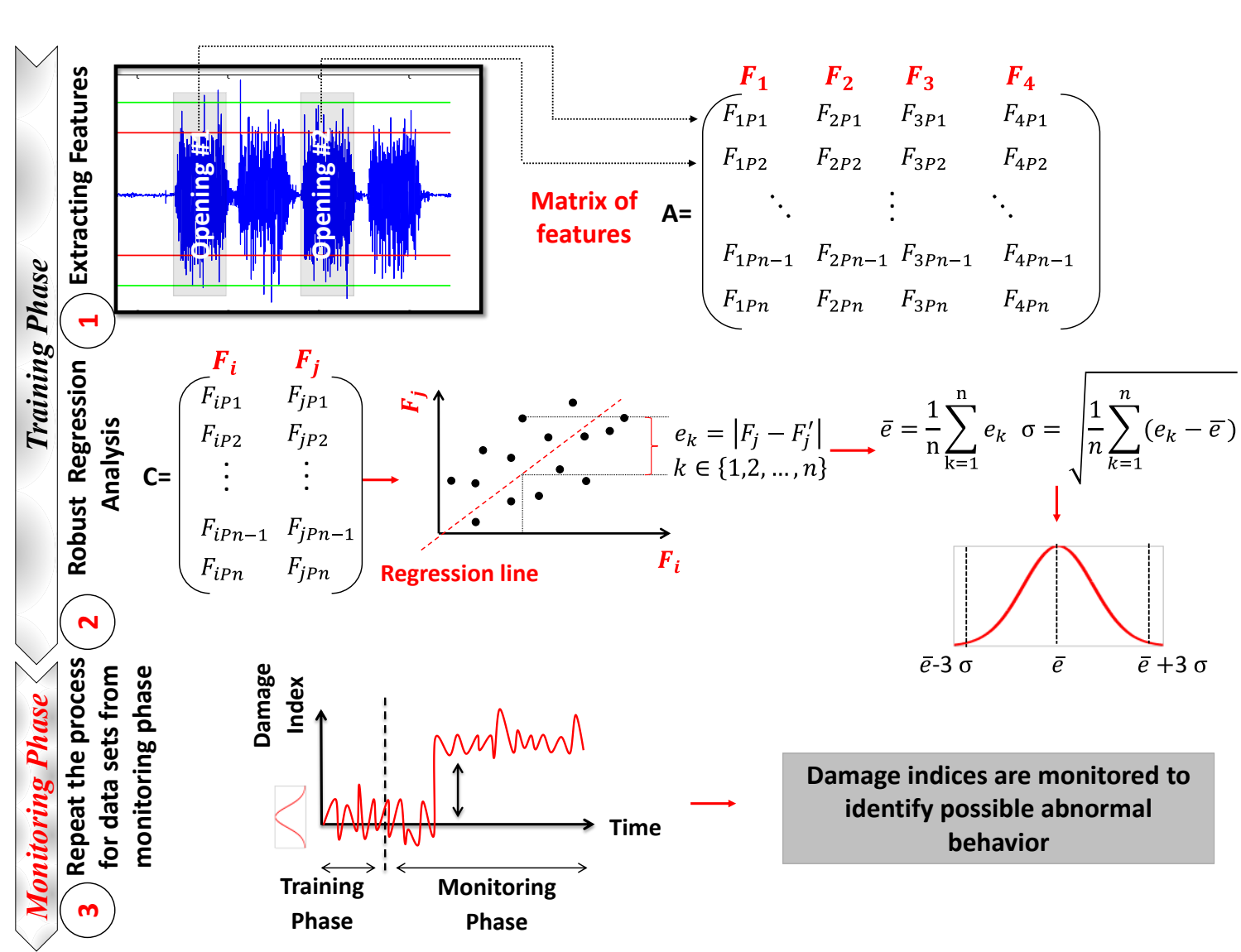

Figure 7- Framework for the proposed algorithm

$F_{i P_{n}}$ indicates $i^{t h}$ feature extracted from $n^{t h}$ opening, as shown in Figs 7 and 8.

\section{Step2: Conducting cross correlation and robust regression analysis on matrix of features}

A correlation threshold is defined as an input to the algorithm and the feature pairs (in matrix $\mathbf{A}$ ) with a correlation coefficient above the threshold are separated and stored in an individual matrix, shown as matrix $\mathbf{C}$ in Figures 7 and 8. Upon identifying all the correlated features, robust regression analysis is performed on each individual feature pairs and the corresponding robust 
regression line is derived (step 2 of Figure 7). For each individual correlated feature pair, error in regression estimation $\left(e_{k}\right)$ is calculated for data points in the training phase and the corresponding underlying distribution of is determined. In fact, this error indicates the difference between the real value of $j^{\text {th }}$ feature $\left(F_{\mathrm{j}}\right)$ and the estimated value based on the regression line $\left(F_{j}^{\prime}\right)$. The error is calculated using Equation 5.

$$
e_{k}=\left|F_{j}-F_{j}^{\prime}\right|
$$

Any functionality issue in mechanical components supposedly affects the structure of the collected data and accordingly the error in prediction model $\left(e_{k}\right)$. Therefore, herein, $\left(e_{k}\right)$ is considered as a damage index.

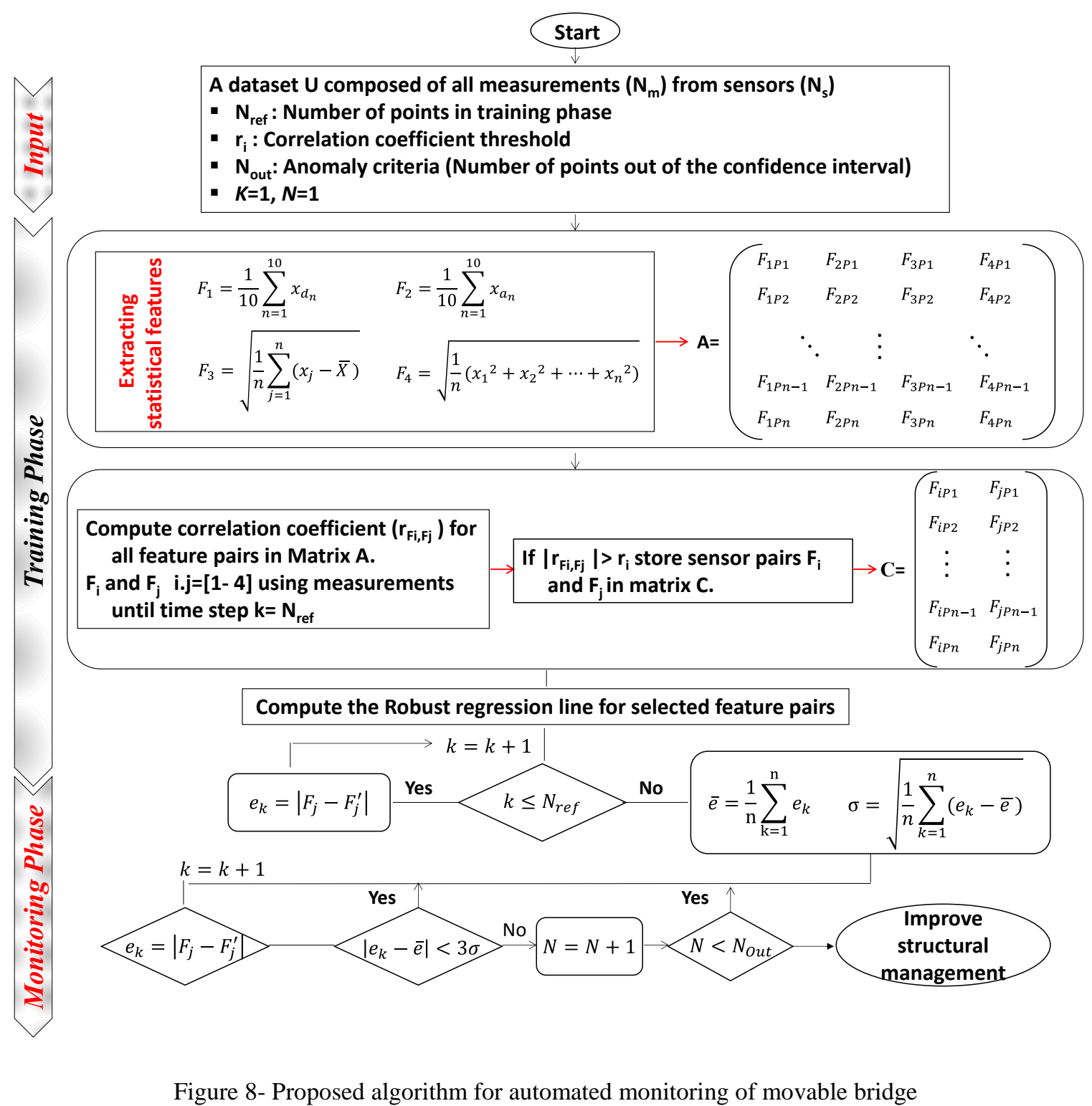

\section{II: Monitoring phase}


Step3: Processing the data from monitoring phase and identify possible abnormal behavior.

These errors are tracked and any significant variation from the corresponding confidence intervals are considered and reported as abnormal behavior in the monitored mechanical components, Equation 6.

$$
\left|e_{k}-\bar{e}\right|>3 \sigma
$$

A flowchart including all steps required in this algorithm is presented in Fig.8. The parameters that should be given as inputs to the algorithms are:

$N_{\text {ref: }}$ Number of data pints in training phase.

As it was discussed earlier, prior to perform the data analysis, data is divided into two portions, training and monitoring. Here, $N_{\text {ref }}$ indicates the number of data points that algorithm should use to learn the underlying distribution of baseline condition.

$r_{i}$ : Correlation threshold.

This parameter is to manage the computational time. Setting threshold too high will significantly reduce the computational time since there will be less number of features to process. However, this will increase the chance of missing abnormal behavior in the monitored structure. On the other hand, if the threshold sets too low then the algorithm consider all the features in the analysis and the computational time significantly increase accordingly. While the best approach to define this threshold is through trial and error, it is suggested to start with the value between 0.3 to 0.4 .

$N_{\text {out }}$ : Anomaly criteria.

Once the number of data points out of confidence interval exceed this amount, the algorithm reports possible abnormal behavior in the monitored structure.

The lower this value is set, the more sensitive the algorithm will be. In fact, setting low $\mathrm{N}$ out will significantly increase the chance of false alarm. On the other hand, the higher the $\mathrm{N}$ out is selected the higher will be the chance of missing abnormal behavior since the sensitivity is decreased. For the purpose of mechanical component monitoring authors suggest 20 consecutive data points as an input for $\mathrm{N}$ out. Therefore, if the calculated damage sensitive feature is out of confidence interval for 20 consecutive steps then the algorithm will raise the red flag.

It should be also noted that if there were enough data sets from damage conditions, then the authors would suggest adding an additional step after monitoring phase to avoid overfitting and be able to classify the damage. This additional step will assure the generalization of the prediction model.

In addition, utilizing RRA algorithm, instead of traditional regression techniques, and also extracting sensitive features based on the 10 highest values will significantly reduce the effect of outliers and noise on the outcomes. In this paper, real data, which inherently incorporate noise, were used. However, effect of outliers and noise can be further explored in future studies. 


\section{EFFICENY OF ALGORITHM FOF DETECTING ABNORMAL BEHAVIOR}

403

404

405

406

407

408

409

410

411

412

413

414

415

416

417

418

419

420

421

422

423

424

425

426

427

428

429

430
As discussed earlier, one of the most common sources of damage to the gearbox is lack of lubrication with oil. In light of this fact, $25 \%$ of oil was extracted from the gearbox at the Sunrise Boulevard Bridge in order to evaluate the efficacy of the proposed algorithm for anomaly detection. The damage indices $\left(e_{k}\right)$ calculated for the gearbox are presented in Figure 9. For the sake of brevity, only the corresponding results for accelerometers 1 and 3 are shown in Figure 9 (there are a total of 6 accelerometers installed on the gearbox).

For accelerometer 1 and 3, as it can be observed from Figure 9, features 1, 2 and 3 are the most correlated and therefore were selected for robust regression analysis. As presented in Figure 9, damage indices (errors in robust regression estimation) are quite effective in detecting the simulated damage, lack of lubrication, in gearbox. Extracting oil from gearbox causes excessive vibration on gearbox during opening and closing. This malfunction, as illustrated in Figure 9, is detected by tracking the proposed damage indices. The underlying distributions of individual damage indices, as shown in Figure 9, are learned during the normal operation (baseline condition). Consequently, any significant variation from these baseline conditions is reported as damage or critical maintenance issue.

As it is observed from Figure 9, the calculated damage indices are confined within the established confidence interval prior to introducing the damage (extracting oil). However, once $25 \%$ of the oil is extracted from the gearbox, the computed damage indices experience an abrupt jump which is observed for all the indices in Figure 9.This reveals the importance of continuous monitoring of the gearbox leakage and the oil level. In fact, any leakage in gearbox (that causes reduction in the oil level) induces additional vibration to the gearbox which might result in major functionality issue for a movable bridge.

Once the extracted oil is replaced, as shown in Figure 9, the damage indices shift back to normal range. 


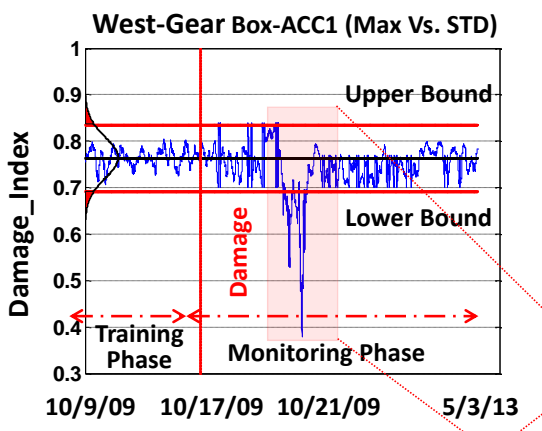

Simulating Damage by Extracting the Oil from the Gearbox

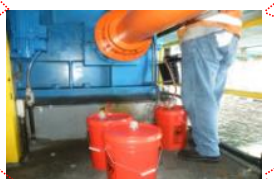

West-Gear Box-ACC 1 (Min Vs. STD)

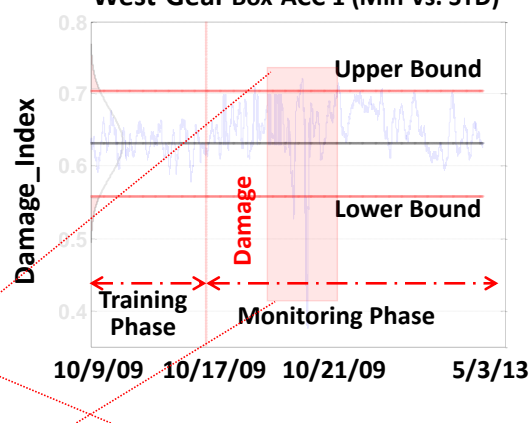

West-Gear Box-ACC3 (Min Vs. STD)

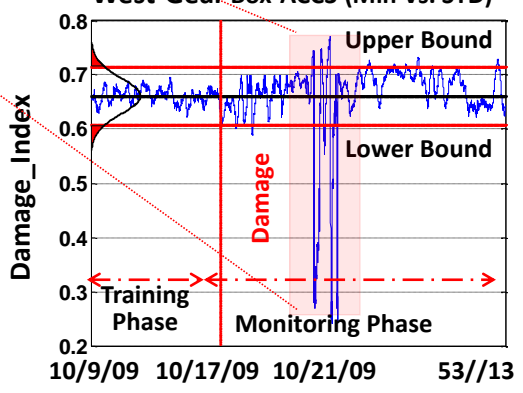

Figure 9- Damage indices calculated based on the data collected from gearbox

Lack of necessary amount of oil to gear meshes and bearings affect the smooth and trouble free operation of gearbox. Any malfunction in gearbox will directly influence the functionality of the motor and open gear, which will subsequently affect the entire bridge operation. Calculating and tracking these damage indices in real-time will provide a great asset management tool for the bridge owners to timely detect and identify any possible abnormal behavior in gearbox. Being timely informed can save both time and budget for the bridge owners. The results shown in this section indicates the proficiency of using this algorithm as a reliable automated framework for continuous monitoring of gearbox in movable bridges.

\subsection{Effect of Extracting Oil from gearbox on motor functionality}

As discussed earlier, motor is monitored using two individual accelerometers. Since motor is directly linked to the gearbox, any malfunction in gearbox is expected to initiate an abnormal behavior for motor operation as well. In order to further investigate this hypothesis, the data collected from the motor accelerometers are also analyzed using the proposed algorithm and the corresponding damage indices are shown in Figure 10. 
West-Motor-ACC1 (RMS Vs. STD)

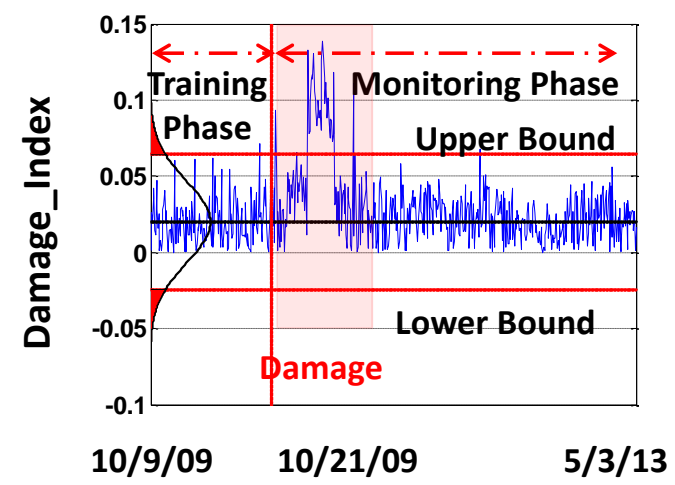

West-Motor-ACC2 (MAX Vs. STD)

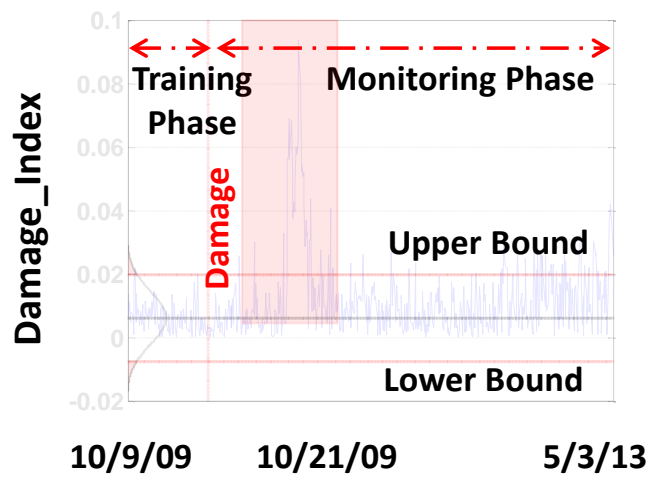

Figure 10- Damage indices calculated based on the data collected from motor

As seen in Figure 10, prior to removing oil from gearbox, damage indices for the motor are confined within the confidence interval. However, once the oil is removed damage indices related to both accelerometers exceed the established confidence intervals. Indeed, as expected, extracting oil from the gearbox causes additional vibration on gearbox and motor since they are both directly linked to each other. Also, another interesting observation is that while for one of the accelerometer abnormal behavior can be detected from the regression line between RMS and STD, for the second accelerometer malfunction is detected from the correlation between Max and STD. This might be due to the location of each accelerometer and unique characteristic of data collected from each individual location. The results confirm the efficiency of the algorithm for detecting excessive vibration on motor as illustrated in Figure 10.

\subsection{Effect of removing bolts on Rack and Pinion functionality}

Two bolts were removed from the North rack and pinion in order to simulate one of the most common maintenance issues (damages) associated with this critical component. The rack and pinion is instrumented with two accelerometers, one in the South and another one in the North. The bolts are removed from the north part of the rack and pinion, as displayed in Figure 11. The corresponding results and damage indices for Rack and Pinion are shown in Figure 10. It is realized that the first damage index (derived based on the North accelerometer) for the rack and pinion shows an abrupt jump due to bolts removal, while there is not any significant variation in the second damage index (derived based on the South accelerometer).

These promising results indicate that the proposed algorithm can also be considered as an effective and reliable technique for identifying the corresponding maintenance issues for rack and pinion. The fact that only the damage index related to the North accelerometer could detect the damage shows that the algorithm is not only cable of detecting malfunctions but can also be utilized for localizing the maintenance issues. 


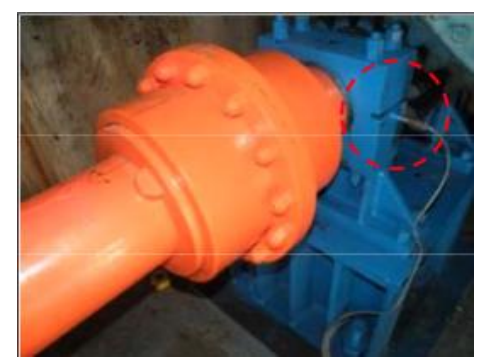

Rack and Pinion_North ACC- (MAX Vs. MIN)

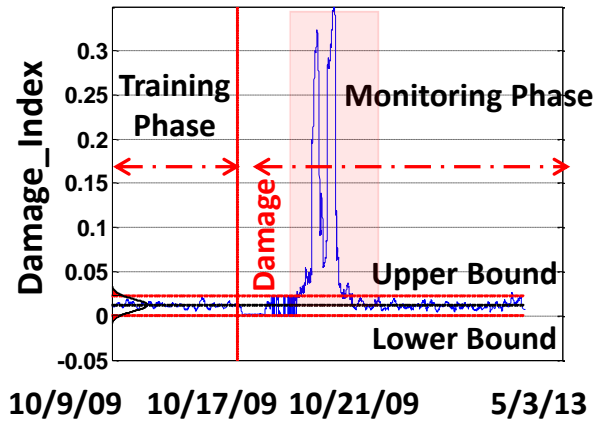

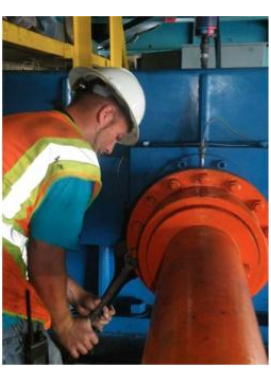

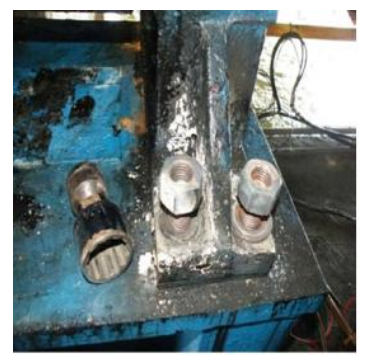

Rack and Pinion_South ACC (MAX Vs. STD)

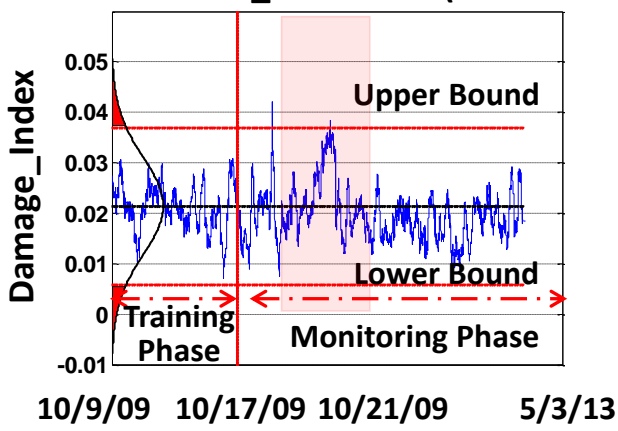

Figure 11- Damage indices calculated based on the data collected from Rack and Pinion

\section{CONCLUSION AND SUMMARY}

A non-parametric (model-free or data-driven) approach is utilized in this study [26]. An algorithm based on RRA is introduced to continuously process the collected massive (big) SHM data and detect any possible malfunction in mechanical components. The presented approach for data analysis consists of a training phase that includes extracting statistical features and conducting cross correlation analysis (CCA) and robust regression analysis (RRA); and then the monitoring phase that includes tracking errors from the prediction models.

Efficacy of the proposed algorithm for abnormal behavior detection is tested using some of the common and critical damage scenarios of movable bridges, which were selected based on the feedback from the DOTs engineers and maintenance personnel. The machinery components are critical elements for the uninterrupted functionality of the movable bridges. As a consequence, the continuous monitoring and assessment of these components are necessary. In this study, the efficiency of implementing SHM system for tracking the condition of the mechanical components including the gearbox, motor and rack and pinion is explored. For that purpose, the Sunrise Blvd. Bridge has been selected and instrumented. Accordingly, the data was collected for about 4 years from all the mechanical and structural components of the bridge.

It is shown that the algorithm is reliable for continuous and automated functionality monitoring of mechanical components of a movable bridge. Four years of SHM data collected from Sunrise Blvd Bridge are utilized to test the efficiency of algorithm under baseline and different common damage scenarios. The algorithm shows very promising results in terms of detecting excessive vibration on gearbox caused by leakage and lack of sufficient oil in gearbox. In addition, 
extracted damage indices are sensitive enough to detect functionality issue related to bolt removal from rack and pinion. Therefore, according to the findings, the introduced algorithm can be utilized for managing and processing massive (big) SHM data collected from mechanical components of a movable bridge.

Such data analysis methods can be designed and implemented on other bridge types for critical locations where any abnormal behavior can be detected and actions can be taken. Especially such non-parametric (model-free or data-driven) approach are quite suitable for monitoring large bridge populations.

\section{ACKNOWLEDMENTS}

The authors acknowledge the support provided by provided by Federal Highway Administration (FHWA) Cooperative Agreement Award DTFH61-07-H-00040 as part of the Exploratory Advanced Research Program as well as Florida Department of Transportation for their support and collaboration. There have been a number of students, researchers and colleagues that need to acknowledged. Specifically, the authors would also like to acknowledge Dr. Mustafa Gul for his contributions of the design and development of the field studies presented in this paper. The senior author also would like to acknowledge the support of National Science Foundation (NSF) \#1463493 for partial funding of the studies presented in this paper. The opinions, findings, and conclusions expressed in this publication are those of the authors and do not necessarily reflect the views of the sponsoring organizations.

\section{References}

[1]. Catbas, F. Necati, Masoud Malekzadeh, and Tung Khuc. "Movable Bridge Maintenance Monitoring." (2013).

[2]. Chin, Roland T., and Charles A. Harlow. "Automated visual inspection: A survey." Pattern Analysis and Machine Intelligence, IEEE Transactions on 6 (1982): 557-573.

[3]. Moore, Mark, et al. Reliability of visual inspection for highway bridges, volume I: Final report. No. FHWA-RD-01-020, 2001.

[4]. Balageas, Daniel, Claus-Peter Fritzen, and Alfredo Güemes, eds. Structural health monitoring. Vol. 493. London: ISTE, 2006.

[5]. Malekzadeh, M., G. Atia, and F. N. Catbas. "Performance-based structural health monitoring through an innovative hybrid data interpretation framework. "Journal of Civil Structural Health Monitoring 5.3 (2015): 287-305.

[6]. Sohn, Hoon, Jerry A. Czarnecki, and Charles R. Farrar. "Structural health monitoring using statistical process control." Journal of Structural Engineering126.11 (2000): 1356-1363.

[7]. Malekzadeh, M., G. Atia, and F. N. Catbas. "A Hybrid Data Interpretation Framework for Automated Performance Monitoring of Infrastructure." Structures Congress 2015. 2015.

[8]. Sohn, Hoon, Jerry A. Czarnecki, and Charles R. Farrar. "Structural health monitoring using statistical process control." Journal of Structural Engineering126.11 (2000): 1356-1363.

[9]. O. AL-Jarrah, P. D. Yoo, S. Muhaidat, G. K. Karagiannidis, and K. Taha, Efficient machine learning for big data: A review, Big Data, Analytics, and High-Performance Computing, 2(3), (2015) 87-93.

[10]. Malekzadeh, Masoud, et al. "Use of FBG sensors to detect damage from large amount of dynamic measurements." Topics on the Dynamics of Civil Structures, Volume 1. Springer New York, 2012. 273-281. 
[11]. Ren, Wei-Xin, and Guido De Roeck. "Structural damage identification using modal data. I: Simulation verification." Journal of Structural Engineering (2002).

[12]. M. Aghagholizadeh, F.N. Catbas, "A Review of Model Updating Methods for Civil Infrastructure Systems", in J. Kruis, Y. Tsompanakis and B.H.V. Topping, (Editors), "Computational Techniques for Civil and Structural Engineering", Saxe-Coburg Publications, Stirlingshire, UK, Chapter 4, pp 83-99, 2015. doi:10.4203/csets.38.4

[13]. Golestani, Behnam, et al. "Nanoclay application to asphalt concrete: Characterization of polymer and linear nanocomposite-modified asphalt binder and mixture." Construction and Building Materials 91 (2015): 32-38.

[14]. Anderson, Marti J. "A new method for non-parametric multivariate analysis of variance." Austral ecology 26.1 (2001): 32-46.

[15]. Malekzadeh, Masoud, et al. "An integrated approach for structural health monitoring using an in-house built fiber optic system and non-parametric data analysis." SMART STRUCTURES AND SYSTEMS 14.5 (2014): 917-942.

[16]. A. Santos, E. Figueiredo, M. F. Silva, C. S. Sales, and J. C. Costa, "Machine learning algorithms for damage detection: Kernel-based approaches," Journal of Sound and Vibration, (2015) 1-16.

[17]. H. Salehi, S. Das, Sh. Chakrabartty, S. Biswas, and R. Burgueno, "Structural assessment and damage identification algorithms using binary data," ASME 2015 Conference on Smart Materials, Adaptive Structures and Intelligent Systems (SMASIS2015), Colorado Springs, Colorado, 2015.

[18]. E. Figueiredo, G. Park, Ch. R. Farrar, K, Worden, and J. Figueiras, "Machine learning algorithms for damage detection under operational and environmental variability," Structural Health Monitoring, 10(6) (2010) 559-572.

[19]. Worden, Keith, and Graeme Manson. "The application of machine learning to structural health monitoring." Philosophical Transactions of the Royal Society of London A: Mathematical, Physical and Engineering Sciences 365.1851 (2007): 515-537.

[20]. Mokhtari, Soroush, et al. "Monitoring Proximity Tunneling Effects Using Blind Source Separation Technique." Structural Health Monitoring, Volume 5. Springer International Publishing, 2014. 111-115.

[21]. Catbas, F. Necati, et al. "Long term bridge maintenance monitoring demonstration on a movable bridge: A framework for structural health monitoring of movable bridges." (2010).

[22]. Laory, Irwanda, Thanh N. Trinh, and Ian FC Smith. "Evaluating two model-free data interpretation methods for measurements that are influenced by temperature." Advanced Engineering Informatics 25.3 (2011): 495-506.

[23]. Rousseeuw, Peter J., and Annick M. Leroy. Robust regression and outlier detection. Vol. 589. John Wiley \& Sons, 2005.

[24]. Posenato, Daniele, et al. "Methodologies for model-free data interpretation of civil engineering structures." Computers \& structures 88.7 (2010): 467-482.

[25]. Malekzadeh, Masoud, and F. Necati Catbas. "A comparative evaluation of two statistical analysis methods for damage detection using fiber optic sensor data. "International Journal of Reliability and Safety 8.2-4 (2014): 135-155.

[26] Catbas, F. N., Gokce, H. B., \& Gul, M. (2012). Nonparametric analysis of structural health monitoring data for identification and localization of changes: Concept, lab, and real-life studies. Structural Health Monitoring, 11(5), 613-626. 